PROCEEDINGS OF THE

AMERICAN MATHEMATICAL SOCIETY

Volume 128, Number 4, Pages 1047-1053

S 0002-9939(99)05145-X

Article electronically published on July 28, 1999

\title{
PSEUDO-DIFFERENTIAL OPERATORS AND MAXIMAL REGULARITY RESULTS FOR NON-AUTONOMOUS PARABOLIC EQUATIONS
}

\author{
MATTHIAS HIEBER AND SYLVIE MONNIAUX \\ (Communicated by Christopher D. Sogge)
}

\begin{abstract}
In this paper, we show that a pseudo-differential operator associated to a symbol $a \in L^{\infty}(\mathbb{R} \times \mathbb{R}, \mathcal{L}(H))$ ( $H$ being a Hilbert space) which admits a holomorphic extension to a suitable sector of $\mathbb{C}$ acts as a bounded operator on $L^{2}(\mathbb{R}, H)$. By showing that maximal $L^{p}$-regularity for the nonautonomous parabolic equation $u^{\prime}(t)+A(t) u(t)=f(t), u(0)=0$ is independent of $p \in(1, \infty)$, we obtain as a consequence a maximal $L^{p}([0, T], H)$-regularity result for solutions of the above equation.
\end{abstract}

\section{INTRODUCTION}

A classical result in the theory of pseudo-differential operators states that an operator associated to a symbol belonging to the class $S^{0}$ acts as a bounded operator on $L^{2}\left(\mathbb{R}^{N}\right.$ ) (see e.g. [12], Ch.VI). It was observed in recent years that pseudodifferential operators with operator-valued symbols (i.e. symbols which take values in the space of bounded linear operators on a Banach space $X$ ) are very useful in proving so called maximal regularity results for autonomous parabolic evolution equations. For details and more information in this direction we refer to [2], [8], [4], [10], [3] and [7]. In this paper we examine maximal $L^{p}$-regularity results for non-autonomous equations of the form

$$
\begin{aligned}
u^{\prime}(t)+A(t) u(t) & =f(t), \quad t \in[0, T], \\
u(0) & =0
\end{aligned}
$$

via the technique of pseudo-differential operators with operator-valued symbols. Since operators $A(t)$ associated to specific boundary value problems arising in applications very often show non-smooth dependence on $t$, we are in particular interested in symbols $a(x, \xi)$ having non-smooth dependence on $x$.

It is one aim of this paper to show, roughly speaking, that a pseudo-differential operator associated to a symbol $a \in L^{\infty}(\mathbb{R} \times \mathbb{R}, \mathcal{L}(H))$, where $H$ is a Hilbert space, which admits a bounded, holomorphic extension to a suitable sector of the complex plane, acts as a bounded operator on $L^{2}(\mathbb{R}, H)$. Considering in particular the symbol $a$ given by $a(t, \tau):=A(t)(i \tau+A(t))^{-1}$ we obtain as a consequence a maximal $L^{2}([0, T], H)$-regularity result for (1.1) provided the family $A(t)_{t \in[0, T]}$ satisfies the so called Acquistapace-Terreni condition. Note that our result generalizes in particular

Received by the editors May 18, 1998.

1991 Mathematics Subject Classification. Primary 35K22, 35S05, 47D06.

(C)2000 American Mathematical Society 
the result of de Simon [5] on $L^{2}(0, T ; H)$-regularity for the autonomous case, i.e. $A(t)=A$ for all $t \in[0, T]$, to equations of the form (1.1).

Observe that we allow that the domains $D(A(t))$ of $A(t)$ may vary with $t \in[0, T]$. Hence maximal regularity results for (1.1) cannot be obtained from those for the autonomous equation by simple perturbation techniques.

We remark that the maximal $L^{2}([0, T] ; H)$-regularity result for $(1.1)$ is the first cornerstone in establishing mixed $L^{p}\left([0, T] ; L^{q}(\Omega)\right)$-estimates $(1<p, q<\infty)$ for equations of the form (1.1). The Calderón-Zygmund theory for operator-valued kernels as developed for instance in [11] allows us to prove that, for arbitrary Banach spaces $X$ and $p \in(1, \infty)$, there is maximal $L^{p}(0, T ; X)$-regularity for $(1.1)$ if and only if there is maximal $L^{2}(0, T ; X)$-regularity for (1.1). Hence we obtain maximal $L^{p}(0, T ; H)$-regularity for $(1.1)$.

In [6] we prove mixed $L^{p}-L^{q}$ estimates for the solution of (1.1) (under suitable assumptions on the heat kernels on the semigroups generated by $A(t)$ ), by interpolating between the $L^{1}-L_{w}^{1}$ result proved in [6] and the $L^{2}-L^{2}$ result stated as Theorem 2.1 below and by applying the fact that the property of maximal $L^{p}$ regularity is independent of $p$. We finally remark that our maximal regularity results may be used to prove existence and uniqueness results for semilinear problems of the form $u^{\prime}(t)+A(t) u(t)=f(t, u(t)), u(0)=0$. For details we refer to $[6]$.

Throughout this paper we denote by $\mathcal{L}(X, Y)$ the space of all bounded linear operators from $X$ to $Y$, whenever $X$ and $Y$ are Banach spaces and by $H$ a Hilbert space. If $A$ is a linear operator in $X$, we denote its domain by $D(A)$, its resolvent set by $\rho(A)$ and its spectrum by $\sigma(A)$. Furthermore, we denote by $\mathcal{S}(\mathbb{R} ; X)$ the space of all rapidly decreasing smooth functions on $\mathbb{R}$. The Fourier transform $\widehat{f}$ of a function $f \in \mathcal{S}(\mathbb{R} ; X)$ is defined by

$$
(\mathcal{F} f)(\xi):=\widehat{f}(\xi):=\int_{\mathbb{R}} e^{-i x \xi} f(x) d x, \quad \xi \in \mathbb{R} .
$$

Finally, we denote by $C$ various constants which may differ from occurrence to occurrence but are always independent of the free variable of a given formula.

\section{Pseudo-Differential operators WITH NON-SMOOTH OPERATOR-VALUED SYMBOLS}

For $\theta \in(0, \pi)$ set $\Sigma_{\theta}:=\{z \in \mathbb{C} \backslash\{0\} ;|\arg z|<\theta\}$. Let $a \in L^{\infty}(\mathbb{R} \times \mathbb{R} ; \mathcal{L}(H))$ and define the pseudo-differential operator

$$
O p(a): \mathcal{S}(\mathbb{R}, H) \rightarrow B C(\mathbb{R}, H)
$$

with operator-valued symbol $a$ by

$$
(O p(a) u)(x):=\frac{1}{2 \pi} \int_{\mathbb{R}} e^{i x \xi} a(x, \xi) \hat{u}(\xi) d \xi, \quad x \in \mathbb{R},
$$

where $\mathcal{S}(\mathbb{R}, H)$ denotes the Schwartz space of rapidly decreasing smooth $H$-valued functions on $\mathbb{R}$.

2.1. Theorem. Let $a \in L^{\infty}(\mathbb{R} \times \mathbb{R}, \mathcal{L}(H))$ and assume that $\xi \mapsto a(x, \xi)$ admits a holomorphic $\mathcal{L}(H)$-valued extension $z \mapsto a(x, z)$ to $\Sigma_{\theta}$ and $-\Sigma_{\theta}$ for some $\theta \in\left(0, \frac{\pi}{2}\right)$ such that $\sup _{z \in \Sigma_{\theta}, z \in-\Sigma_{\theta}} \sup _{x \in \mathbb{R}}\|a(x, z)\|_{\mathcal{L}(H)}<\infty$. Then the operator $O p(a)$, initially defined on $S(\mathbb{R}, H)$, extends to a bounded operator on $L^{2}(\mathbb{R}, H)$. 
Proof. Let $\alpha:=\frac{\sin \theta}{2}$ and set $R:=1+\frac{\alpha}{2}$. Choose $\varphi \in C_{c}^{\infty}(\mathbb{R})$ with $\operatorname{supp} \varphi \subset$ $\left(R^{-1}, R\right)$ such that

$$
\int_{\mathbb{R}} \frac{\varphi^{2}(\tau)}{|\tau|} d \tau=1
$$

For $u \in S(\mathbb{R}, H)$ we then have

$$
(O p(a) u)(x)=\frac{1}{2 \pi} \int_{\mathbb{R}} \int_{\mathbb{R}} e^{i x \xi} a(x, \xi) \varphi^{2}\left(\frac{\xi}{\tau}\right) \hat{u}(\xi) d \xi \frac{1}{|\tau|} d \tau .
$$

Furthermore, let $\Gamma:=\{z \in \mathbb{C} ;|z-1|=\alpha\}$ be positively oriented. By Cauchy's theorem we have

$$
a(x, \xi)=\frac{1}{2 \pi i} \int_{\Gamma} \frac{a(x, \tau z)}{z-\xi / \tau} d z \quad(x \in \mathbb{R})
$$

for those $(\xi, \tau) \in \mathbb{R} \times \mathbb{R}$ satisfying $\varphi\left(\frac{\xi}{\tau}\right) \neq 0$. Inserting this in (2.1) we obtain by Fubini's theorem

$$
(O p(a) u)(x)=\frac{1}{2 \pi i} \int_{\Gamma} \int_{\mathbb{R}} \int_{\mathbb{R}} \frac{1}{2 \pi} e^{i x \xi} \frac{\varphi^{2}\left(\frac{\xi}{\tau}\right)}{z-\frac{\xi}{\tau}} a(x, \tau z) \hat{u}(\xi) d \xi \frac{d \tau}{|\tau|} d z .
$$

Setting $g_{z, \tau}(\xi):=\frac{\varphi\left(\frac{\xi}{\tau}\right)}{z-\frac{\xi}{\tau}}, h_{\tau}(\xi):=\varphi\left(\frac{\xi}{\tau}\right)$ and denoting the inner integral above by $I_{\tau, z} u$ we obtain

$$
\left(I_{\tau, z} u\right)(x)=\left(\mathcal{F}^{-1}\left(g_{z, \tau}\right) * \mathcal{F}^{-1}\left(h_{\tau}\right) * a(x, \tau z) u\right)(x), \quad x \in \mathbb{R} .
$$

Since $\operatorname{supp} \varphi \subset\left(R^{-1}, R\right)$ it follows from Plancherel's theorem that

$$
\left(I_{\tau, z} u, I_{\rho, z} u\right)_{L^{2}(\mathbb{R}, H)}=0
$$

provided $\frac{\rho}{\tau} \in \mathbb{R} \backslash\left\{\left(R^{-2}, R^{2}\right)\right\}$. In order to estimate $O p(a) u$ in $L^{2}(\mathbb{R}, H)$ notice that

$$
\|O p(a) u\|_{L^{2}(\mathbb{R}, H)} \leq \frac{1}{2 \pi} \int_{\Gamma}\left\|H_{z} u\right\|_{L^{2}(\mathbb{R}, H)} d z,
$$

where $H_{z} u:=\int_{\mathbb{R}} I_{\tau, z} u \frac{d \tau}{|\tau|}$ is understood as an inproper integral in $L^{2}(\mathbb{R}, H)$. It follows from (2.2) and the Cauchy-Schwarz inequality that

$$
\begin{aligned}
\left\|H_{z} u\right\|_{L^{2}(\mathbb{R}, H)}^{2} & =\int_{\mathbb{R}} \int_{\mathbb{R}}\left(I_{\tau, z} u, I_{\rho, z} u\right)_{L^{2}(\mathbb{R}, H)} \frac{d \rho}{|\rho|} \frac{d \tau}{|\tau|} \\
& =\int_{R^{-2}} \int_{\mathbb{R}}\left(I_{\tau, z} u, I_{\tau \rho, z} u\right)_{L^{2}(\mathbb{R}, H)} \frac{d \tau}{|\tau|} \frac{d \rho}{|\rho|} \\
& \leq \int_{R^{-2}}^{R_{\mathbb{R}}^{2}}\left\|I_{\tau, z} u\right\|_{L^{2}(\mathbb{R}, H)}^{2} \frac{d \tau}{|\tau|} \frac{d \rho}{|\rho|} \\
& =4 \log R \int_{\mathbb{R}}\left\|I_{\tau, z} u\right\|_{L^{2}(\mathbb{R}, H)}^{2} \frac{d \tau}{|\tau|} .
\end{aligned}
$$


Observe that by Plancherel's theorem we have

$$
\left\|I_{\tau, z} u\right\|_{L^{2}(\mathbb{R}, H)} \leq \sup _{x \in \mathbb{R}}\|a(x, \tau z)\|_{\mathcal{L}(H)} \sup _{\eta \in \mathbb{R}}\left|\frac{\varphi(\eta)}{z-\eta}\right|\left\|h_{\tau} \hat{u}\right\|_{L^{2}(\mathbb{R}, H)} .
$$

Therefore there exists a constant $C>0$ such that

$$
\begin{aligned}
\left\|H_{z} u\right\|_{L^{2}(\mathbb{R}, H)}^{2} & \leq C \int_{\mathbb{R}}\left\|h_{\tau} \hat{u}\right\|_{L^{2}(\mathbb{R}, H)}^{2} \frac{d \tau}{|\tau|} \\
& =C \int_{\mathbb{R}} \int_{\mathbb{R}}\left|\varphi\left(\frac{\xi}{\tau}\right)\right|^{2} \frac{d \tau}{|\tau|}\|\hat{u}(\xi)\|_{H}^{2} d \xi=C\|u\|_{L^{2}(\mathbb{R}, H)}^{2} .
\end{aligned}
$$

Combining this estimate with (2.3) it follows that

$$
\|O p(a) u\|_{L^{2}(\mathbb{R}, H)} \leq C\|u\|_{L^{2}(\mathbb{R}, H)}
$$

for $u \in S(\mathbb{R}, H)$ and by density for all $u \in L^{2}(\mathbb{R}, H)$.

\section{MAXimal REGUlarity FOR NON-AUTONOMOUS PARABOLIC EQUATIONS}

Let $T>0$ and let $(A(t))_{t \in[0, T]}$ be a family of densely defined linear operators in $X$ satisfying the following two assumptions:

A1) There exists $\theta \in(0, \pi / 2)$ such that $\sigma(A(t)) \subset \Sigma_{\theta}$ for all $t \in[0, T]$ and for $\varphi \in(\theta, \pi)$ there exists $M>0$ such that

$$
\left\|(\lambda-A(t))^{-1}\right\|_{\mathcal{L}(X)} \leq \frac{M}{1+|\lambda|}, \quad t \in[0, T], \lambda \in \mathbb{C} \backslash \Sigma_{\varphi} .
$$

A2) There exist constants $\alpha, \beta \in[0,1], \alpha<\beta, \omega \in(\theta, \pi / 2), c>0$ such that

$$
\left\|A(t)(\lambda-A(t))^{-1}\left(A(t)^{-1}-A(s)^{-1}\right)\right\|_{\mathcal{L}(X)} \leq c \frac{|t-s|^{\beta}}{1+|\lambda|^{1-\alpha}}
$$

for $s, t \in[0, T], \lambda \in \mathbb{C} \backslash \Sigma_{\omega}$.

We remark that the above conditions A1), A2) on $A(t)$ were introduced and investigated by Acquistapace, Terreni [1] and Yagi [13] in order to construct the evolution operator associated with $A(t), t \in[0, T]$.

Let $1<p<\infty$ and $f:[0, T] \rightarrow X$ be a function. We consider the following non-autonomous initial value problem:

$$
\begin{aligned}
u^{\prime}(t)+A(t) u(t) & =f(t), \quad t \in[0, T], \\
u(0) & =0 .
\end{aligned}
$$

The family $\{A(t), t \in[0, T]\}$ is said to belong to the class $M R(p, X)$ and we say that there is maximal $L^{p}$ regularity for (3.1) if for each $f \in L^{p}(0, T ; X)$ there exists a unique

$$
u \in W^{1, p}(0, T ; X) \quad \text { with } \quad t \mapsto A(t) u(t) \in L^{p}(0, T ; X)
$$

satisfying $(3.1)$ in the $L^{p}(0, T ; X)$-sense.

The following two theorems are the main results of this section.

3.1. Theorem. Let $X$ be a Banach space, $T>0$, and assume that $\{A(t), t \in$ $[0, T]\}$ satisfies A1) and A2). Suppose that there exists $p \in(1, \infty)$ such that the family $\{A(t), t \in[0, T]\}$ belongs to the class $M R(p, X)$. Then $\{A(t), t \in[0, T]\}$ belongs to $M R(q, X)$ for all $q \in(1, \infty)$. 
3.2. Theorem. Let $H$ be a Hilbert space, $1<p<\infty, T>0$ and assume that $\{A(t), t \in[0, T]\}$ satisfies A1) and A2). Then $\{A(t), t \in[0, T]\}$ belongs to $M R(p, H)$.

We start the proof of the two theorems above with the following observation. It follows from the results in [1], [9] that if $u$ is a solution of (3.1), then $u$ fulfills

$$
\begin{aligned}
A(t) u(t)= & \int_{0}^{t} A(t)^{2} e^{-(t-s) A(t)}\left(A(t)^{-1}-A(s)^{-1}\right) A(s) u(s) d s \\
+ & \int_{0}^{t} A(t) e^{-(t-s) A(t)} f(s) d s
\end{aligned}
$$

for $t \in[0, T]$. For the time being let $q \in(1, \infty)$ and define the operator $Q \in$ $\mathcal{L}\left(L^{q}(0, T ; X)\right)$ by

$$
(Q g)(t):=\int_{0}^{t} A(t)^{2} e^{-(t-s) A(t)}\left(A(t)^{-1}-A(s)^{-1}\right) g(s) d s, \quad t \in[0, T] .
$$

The results in [1] and [9] imply that $\|Q\|_{\mathcal{L}\left(L^{q}(0, T ; X)\right)} \leq 1 / 2$ provided the constant $c$ in A2) is sufficiently small. Observe, however, that the family $\{A(t), t \in[0, T]\}$ belongs to the class $M R(q ; X)$ if and only if this holds true for $\{A(t)+K, t \in[0, T]\}$, where $K$ denotes an arbitrary constant. Hence, there is no loss of generality in choosing $c$ as small as we want. It follows that the operator $I d-Q$ is invertible in $L^{q}(0, T ; X)$. Moreover, by $(3.2)$ we know that

$$
(I d-Q) A(\cdot) u=S f, \quad \text { where } \quad(S f)(t):=\int_{0}^{t} A(t) e^{-(t-s) A(t)} f(s) d s
$$

provided $u$ is a solution of (3.1). Summarizing, we proved the following fact.

3.3. Proposition. The family $\{A(t), t \in[0, T]\}$ belongs to the class $M R(q ; X)$ if and only if $S$ acts a bounded operator on $L^{q}(0, T ; X)$.

We are now in the position to prove Theorem 3.1.

Proof of Theorem 3.1. By assumption and Proposition 3.1 we know that $S$ acts boundedly on $L^{p}(0, T ; X)$. In order to show that $S$ is bounded on $L^{q}(0, T ; X)$ for $q \in(1, \infty)$, if suffices to verify (see [11], Theorems III.1.2, III.1.3) that

$$
\begin{gathered}
\sup _{s, s^{\prime} \in(0, T)} \int_{\left|s-s^{\prime}\right| \leq \frac{|t-s|}{2}}\left\|k(t, s)-k\left(t, s^{\prime}\right)\right\| d t<\infty, \\
\sup _{s, s^{\prime} \in(0, T)} \int_{\left|s-s^{\prime}\right| \leq \frac{|t-s|}{2}}\left\|k(s, t)-k\left(s^{\prime}, t\right)\right\| d t<\infty
\end{gathered}
$$

where $k(t, s):=A(t) e^{-(t-s) A(t)} 1_{(0, t)(s)}$. 
To this end, note that for $s, s^{\prime} \in(0, T)$ we have

$$
\begin{aligned}
& \int_{\left|s-s^{\prime}\right| \leq \frac{1}{2}|t-s|}\left\|k(t, s)-k\left(t, s^{\prime}\right)\right\| d t \\
= & \int_{\left|s-s^{\prime}\right| \leq \frac{1}{2}(t-s)}\left\|A(t) e^{-(t-s) A(t)} 1_{(0, t)}(s)-A(t) e^{-\left(t-s^{\prime}\right) A(t)} 1_{(0, t)}\left(s^{\prime}\right)\right\| d t \\
= & \int_{\left|s-s^{\prime}\right| \leq \frac{1}{2}(t-s)}\left\|\int_{s}^{s^{\prime}} A(t)^{2} e^{-(t-\sigma) A(t)} d \sigma\right\| d t \\
\leq & \int_{\left|s-s^{\prime}\right| \leq \frac{1}{2}(t-s)}\left|\int_{s}^{s^{\prime}} \frac{M}{(t-\sigma)^{2}} d \sigma\right| d t=M \int_{\left|s-s^{\prime}\right| \leq \frac{1}{2}(t-s)}\left|\frac{1}{t-s}-\frac{1}{t-s^{\prime}}\right| d t \\
< & \infty .
\end{aligned}
$$

Moreover, for $s, s^{\prime} \in(0, T)$, we have

$$
\begin{aligned}
& \int_{\left|s-s^{\prime}\right| \leq \frac{1}{2}|t-s|}\left\|k(s, t)-k\left(s^{\prime}, t\right)\right\| d t \\
& =\quad \int_{\left|s-s^{\prime}\right| \leq \frac{1}{2}|t-s|}\left\|A(s) e^{-(s-t) A(s)} 1_{s \geq t}-A\left(s^{\prime}\right) e^{-\left(s^{\prime}-t\right) A\left(s^{\prime}\right)} 1_{s^{\prime} \geq t}\right\| d t \\
& \leq \quad \int_{\left|s-s^{\prime}\right| \leq \frac{1}{2}|t-s|}\left\|A(s) e^{-(s-t) A(s)}-A(s) e^{-\left(s^{\prime}-t\right) A(s)}\right\| d t \\
& +\int_{\left|s-s^{\prime}\right| \leq \frac{1}{2}|t-s|}\left\|A(s) e^{-\left(s^{\prime}-t\right) A(s)}-A\left(s^{\prime}\right) e^{-\left(s^{\prime}-t\right) A\left(s^{\prime}\right)}\right\| d t \\
& \leq \quad \int_{\left|s-s^{\prime}\right| \leq \frac{1}{2}|t-s|}\left\|\int_{s}^{s^{\prime}} A(s)^{2} e^{-(\sigma-t) A(s)} d \sigma\right\| d t \\
& +\int_{\left|s-s^{\prime}\right| \leq \frac{1}{2}|t-s|}\left\|\frac{1}{2 \pi i} \int_{\Gamma_{\theta}} \lambda e^{-\left(s^{\prime}-t\right) \lambda}\left((\lambda-A(s))^{-1}-\left(\lambda-A\left(s^{\prime}\right)\right)^{-1}\right) d \lambda\right\| d t \\
& \leq \quad \int_{\left|s-s^{\prime}\right| \leq \frac{1}{2}|t-s|}\left|\int_{s}^{s^{\prime}} \frac{M}{|t-\sigma|^{2}} d \sigma\right| d t \\
& +\int_{\left|s-s^{\prime}\right| \leq \frac{1}{2}|t-s|}\left(\frac{1}{\pi} \int_{0}^{\infty} r e^{-\left(s^{\prime}-t\right) r \cos \theta} \frac{c(M+1)\left|s-s^{\prime}\right|^{\beta}}{(1+r)^{1-\alpha}} d r\right) d t \\
& <\infty \text {. }
\end{aligned}
$$

The proof is complete. 
Proof of Theorem 3.2. Observe that the symbol $a$ defined by

$$
a(t, \tau):= \begin{cases}A(0)(i \tau+A(0))^{-1}, & t<0, \\ A(t)(i \tau+A(t))^{-1}, & t \in[0, T], \\ A(T)(i \tau+A(T))^{-1}, & t>T,\end{cases}
$$

satisfies, thanks to A1), the assumptions of Theorem 2.1. Hence it follows from this theorem and Proposition 3.3 that the family $\{A(t), t \in[0, T]\}$ belongs to the class $M R(2 ; H)$. Theorem 3.1 implies now the assertion.

\section{REFERENCES}

[1] Acquistapace, P., Terreni, B.: A unified approach to abstract linear nonautonomous parabolic equations. Rend. Sem. Mat. Univ. Padova 78, (1987), 47-107. MR 89e:34099

[2] Amann, H.: Linear and Quasilinear Parabolic Problems. Birkhäuser, Basel, (1995). MR 96g:34088

[3] Amann, H.: Operator-valued Fourier multipliers, vector-valued Besov spaces, and applications. Math. Nachr. 186 (1997), 5-56. MR 98h:46033

[4] Coulhon,T., Lamberton, D.: Régularité $L^{p}$ pour les équations d'évolution. In: B. Beauzaumy, B. Maurey, G. Pisier (eds.): Seminaire d'Analyse Fonctionelle 1984/85; Publ. Math. Univ. Paris VII , 26,(1986), 155-165. MR 89e:34100

[5] DE Simon, L.: Un applicazione della teoria degli integrali singolari allo studio delle equazioni differenziali lineari astratta del primo ordine. Rend. Sem. Mat. Univ. Padova, 34 (1964), $547-558$.

[6] Hieber, M., Monniaux, S.: Heat-Kernels and Maximal $L^{p}-L^{q}$ - Estimates: The NonAutonomous Case. Preprint, 1998.

[7] Hieber, M., Prüss, J.: Heat kernels and maximal $L^{p}-L^{q}$ estimates for parabolic evolution equations. Commun. in Partial Differential Equations 22, (1997), 1647-1669. MR 98k:34096

[8] Lunardi, A.: Analytic Semigroups and Optimal Regularity in Parabolic Equations. Birkhäuser, Basel, (1995). MR 96e:47039

[9] Monniaux, S., Rhandi, A.: Semigroup methodes to solve non-autonomous evolution equations. Semigroup Forum, to appear.

[10] PRÜss, J.: Evolutionary Integral Equations and Applications. Birkhäuser, Basel, (1993). MR 94h:45010

[11] Rubio de Francia, J.L., Ruiz, F.J., Torrea, J.L.: Calderón-Zygmund theory for operatorvalued kernels. Adv. Math. 62, (1986), 7-48. MR 88f:42035

[12] Stein, E.M.: Harmonic Analysis: Real-Variables Methods, Orthogonality and Oscillartory Integrals. Princeton University Press, Princeton, (1993). MR 95c:42002

[13] YAGI, A.: Parabolic evolution equations in which coefficients are the generators of infinitely differentiable semigroups, II. Funk. Ekvacioj 33,(1990), 139-150. MR 91h:47039

Mathematisches Institut I, Englerstr. 2, Universität Karlsruhe, D-76128 Karlsruhe, Germany

E-mail address: matthias.hieber@math.uni-karlsruhe.de

Abteilung Mathematik V, Universität Ulm, D-89069 Ulm, Germany

E-mail address: monniaux@mathematik.uni-ulm.de

Current address: Laboratoire de Mathématiques Fondamentales et Appliquées, Centre de Saint-Jérôme, Case Cour A, Avenue Escadrille Normandie-Niemen, 13397 Marseille Cédex 20, France

E-mail address: sylvie.monniaux@math.u-3mrs.fr 